\title{
Proposta de abordagem para refinamento de pesquisa bibliográfica
}

\begin{abstract}
Eduardo Amadeu Dutra Moresi ${ }^{1}$ e Isabel Pinho ${ }^{2}$
1 Programa de Mestrado e Doutorado em Educação da Universidade Católica de Brasília, Brasil | moresi@p.ucb.br | https://orcid.org/0000-0001-6058-3883

${ }^{2}$ Universidade de Aveiro, Portugal | isabelpinho@ua.pt | http://orcid.org/0000-0003-17148979

Resumo: O mapeamento científico tem uma longa tradição e várias abordagens foram propostas, como a análise de citações de periódicos, a análise de cocitações, o acoplamento bibliográfico e a análise de coocorrência de palavras. Este artigo explora a análise de coocorrência de palavras-chave dos autores para realizar o refinamento de pesquisas bibliográficas. É proposta uma abordagem que parte de uma pesquisa bibliográfica mais abrangente e usa a análise de rede de coocorrência de palavras-chave para identificar pares de termos que possam ser utilizados na construção de uma nova expressão de busca para refinar a pesquisa inicial. São apresentados dois exemplos de como utilizar a abordagem proposta. Foi realizada uma pesquisa sobre o tema Covid-19, selecionando as seguintes opções de refinamento: escolha da grande área de Ciências Sociais e dos artigos que tratavam de educação. Em relação aos tipos de documentos, foram incluídos os artigos em periódicos e conferências, além dos documentos de revisão. Em seguida, são exemplificadas as duas alternativas propostas para o refinamento da pesquisa bibliográfica inicial. Concluise que a abordagem proposta evidencia os principais conceitos associados ao tema pesquisado e também pode ser empregado, de forma exploratória, para identificar conceitos pertinentes a uma necessidade de informações sobre um domínio temático.
\end{abstract}

Palavras-chave: Pesquisa Bibliográfica; Bibliometria; Rede de Coocorrência; Covid-19; Educação Superior.

\section{Proposed Approach for Refinement of Bibliographic Research}

\begin{abstract}
Scientific mapping has a long tradition and several approaches have been proposed, such as journal citation analysis, co-citation analysis, bibliographic coupling and words co-occurrence analysis. This paper explores the authors' keywords co-occurrence analysis to refine bibliographic research. An approach is proposed that starts from a more comprehensive bibliographic search and uses the keyword co-occurrence network analysis to identify pairs of keywords that can be used in the construction of a new search expression to refine the initial search. Two examples of how to use the proposed method are presented. A search was carried out on the Covid-19 keyword, selecting the following refinement options: choosing the subject area of Social Sciences and the documents dealing with education. Regarding the types of documents, papers in journals and conferences were included, in addition to the review documents. Then, the two alternatives proposed for the refinement of the initial bibliographic research are exemplified. It is concluded that the proposed approach highlights the main concepts associated with the researched theme and can also be used, in an exploratory way, to identify concepts pertinent to an information need on a thematic domain
\end{abstract}

Keywords: Bibliographic Research; Bibliometric, Co-occurrence Network; Higher Education.

\section{Introdução}

O mapeamento científico tem uma longa tradição que começou com o trabalho de Small (1973) e de outros como Marshakova (1973) e Kessler (1963). Desde então, vários métodos foram propostos, como a análise de citações de periódicos, a análise de cocitações, o acoplamento bibliométrico e a análise de coocorrência de palavras. Com base na coocorrência de pares de palavras, este tipo de análise busca extrair os temas da ciência e detectar as suas relações diretamente com o conteúdo dos textos.

Um estudo de Ding, Chowdhury \& Foo (2001) mostrou a viabilidade da análise de coocorrência de palavras como uma abordagem eficaz para extrair padrões e identificar tendências em grandes corpora onde os textos coletados são do mesmo domínio ou subdomínio e são divididos em quantidades aproximadamente equivalentes para diferentes períodos. 
Besselaar \& Heimeriks (2006) propuseram um método em que as palavras do título são utilizadas como indicadores do conteúdo de um tópico de pesquisa e as referências citadas como o contexto no qual as palavras obtêm seu significado. Esse método apresenta várias vantagens, como alta cobertura de publicações.

Ravikumar, Agrahari \& Singh (2015) estudaram 959 artigos em texto completo para explorar a estrutura intelectual da cienciometria, no período de 2005-2010, usando mineração de textos e análise de coocorrência de palavras. As tendências e padrões da cienciometria, na revista Scientometrics, foram revelados medindo-se a força de associação de palavras-chave selecionadas que representam os conceitos e ideias produzidos no campo da cienciometria.

A análise de coocorrência de palavras (Callon, Courtial, Turner, \& Bauin, 1983) é uma técnica de análise de conteúdo que usa as palavras em documentos para estabelecer relações e construir uma estrutura conceitual do domínio. A ideia subjacente ao método é que, quando as palavras frequentemente coocorrem em documentos, isso significa que os conceitos por trás dessas palavras estão intimamente relacionados. É o único método que utiliza o conteúdo real dos documentos para construir uma medida de similaridade, enquanto os demais conectam os documentos indiretamente por meio de citações ou coautorias.

O resultado da análise de coocorrência de palavras é uma rede de temas e suas relações que representam o espaço conceitual de um campo. Este mapa semântico ajuda a entender sua estrutura cognitiva (Börner, Chen, \& Boyack, 2003). Uma série de mapas produzidos para diferentes períodos de tempo pode rastrear as mudanças neste espaço conceitual (Coulter, Monarch, \& Konda, 1998). A análise de coocorrência de palavras pode ser aplicada a títulos de documentos, palavras-chave, resumos ou textos completos. A unidade de análise é um conceito, não um documento, autor ou periódico.

Diferentes maneiras de realizar análises bibliométricas têm sido propostas usando as métricas, layouts e recursos de visualização de análise de redes (AR). As métricas de AR nos permitem categorizar as redes bibliométricas. Em relação à topologia, Gan \& Wang (2015) e Choi, Yi \& Lee (2011) observaram que as redes de coocorrência de palavraschave seguem uma distribuição de lei de potência ou Lei de Zipf. Assim, as redes que se enquadram na suposição de vinculação preferencial não têm escala, ou seja, novos nós tenderão a se conectar preferencialmente com aqueles altamente conectados. Assim, as palavras-chave altamente frequentes são mais prováveis de serem usadas junto com aquelas que foram recém-introduzidas.

Wood \& Khan (2015) e Khan \& Wood (2015) usaram diferentes medidas, tais como centralidades de grau, de intermediação e de autovetor em redes de coocorrência de palavras, ao analisarem artigos que tratam de negociações comerciais internacionais e domínios de gestão de tecnologia da informação, respectivamente. Lee et al. (2018) usaram as palavras-chave com alta centralidade para mostrar a estrutura geral da exploração espacial humana. Zhao, Mao, \& Lu (2018) classificaram as palavras-chave com base em várias métricas de nós em redes de coocorrência de palavras de três disciplinas diferentes e estabeleceram a frequência das palavras-chave como um método eficaz para identificar temas importantes.

Mais recentemente, Luo, Dai, \& Huang (2021) recuperaram 979 documentos no campo da visualização de informações do patrimônio cultural, entre 2000 e 2020, na base Web Of Science. Com as palavras-chave, como unidade de análise, e coocorrência, como tipo de análise, eles identificaram os tópicos de pesquisa com maiores destaques e os caminhos de evolução em diferentes períodos.

Em suma, a análise de coocorrência de palavras estuda as relações entre as palavraschave usadas para revelar a estrutura e o desenvolvimento de cada área do conhecimento, pesquisa ou metodologia particular. Contudo não foram identificadas aplicações de análise de redes de coocorrência de palavras para o refinamento de pesquisas bibliográficas. Portanto, a questão de pesquisa explorada neste trabalho é: como usar a análise de redes de coocorrência de palavras-chave para refinar a pesquisa bibliográfica sobre um tema? 


\section{A proposta de Abordagem para Refinamento da Pesquisa Bibliográfica}

Este trabalho tem como objetivo apresentar uma análise de coocorrência de palavraschave para refinar a pesquisa bibliográfica de um tema. Para isso, está sendo proposta uma abordagem para alcançar este objetivo.

A abordagem proposta segue as seguintes etapas:

1. Realizar pesquisa bibliográfica definindo uma expressão de busca que atenda o foco da pesquisa;

2. Salvar os metadados em formato CSV, se a pesquisa for realizada na base Scopus, ou TXT, para a base Web of Science;

3. Importar os metadados pelo software VOSviewer (Van Eck \& Waltman, 2021):

- Escolher a unidade de análise palavras-chave dos autores e o tipo de análise coocorrência;

- No mínimo de ocorrência de uma palavra-chave, selecionar uma quantidade que obtenha uma rede com mais de 1.000 palavras-chave ${ }^{1}$;

- Gerar a rede de coocorrência de palavras-chave;

- Construir o tesauros para o controle do vocabulário;

- Gerar a rede de coocorrência de palavras-chave com o tesauros; salvar a rede em formato GML;

4. Importar a rede no software Gephi (Bastian, Heymann, \& Jacomy, 2009) e calcular as métricas de análise de redes - grau médio, classes de modularidade e centralidade de autovetor;

5. No Laboratório de Dados do Gephi selecionar a opção de arestas. A análise das arestas (pares de palavras-chave) indicará a combinação de termos para construir a expressão de busca para a nova pesquisa bibliográfica, que poderá ter as seguintes alternativas:

- Analisar os resultados das arestas com maiores pesos, que irão incluir as palavras-chave com maior grau na rede, e escolher os pares de palavraschave pertinentes ao refinamento desejado². Contudo, a construção da expressão de busca deve considerar o tema central da pesquisa. Caso o par de palavras-chave não inclua a expressão de busca que deu origem à pesquisa, esta deverá ser incluída com um conector AND;

- Escolher um conceito representado por uma palavra-chave e selecionar os pares de arestas que atendam refinamento desejado3;

6. Após a definição da alternativa acima, será construída uma nova expressão de busca combinando as palavras-chave de uma aresta com o operador lógico AND. Esta etapa será executada de forma recursiva, onde a nova expressão de busca será consultada na base bibliográfica e identificada a quantidade de documentos recuperados. Cada novo par de palavras-chave será combinado usando o operador lógico OR, conforme mostrado na Tabela $1^{4}$, e consultado na base de pesquisa bibliográfica.

${ }^{1} \mathrm{O}$ ideal é usar a ocorrência de 1 vez. Contudo, se o quantitativo de palavras-chave for elevado, tipicamente superior a 5.000, o processamento dependerá da capacidade da máquina e das limitações do software.

2 Algumas palavras-chave, apesar de terem importância na rede, não são pertinentes ao refinamento desejado. Por isso, não deverão ser consideradas na nova expressão de busca.

${ }^{3}$ Ver comentário anterior.

${ }^{4}$ Cada linha da Tabela 1 corresponderá ao resultado da consulta da expressão de busca na base de pesquisa bibliográfica. 
A pesquisa poderá ser encerrada quando a quantidade de documentos recuperada alcançar a saturação, ou seja, o acréscimo de documentos for mínimo;

7. Após alcançar a saturação, os metadados da pesquisa bibliográfica serão salvos e importados pelo VOSviewer para gerar as redes desejadas e exportados em formato GML;

8. Importar o GML pelo Gephi, calcular as métricas de redes e extrair as informações necessárias à interpretação dos resultados.

No próximo item será apresentado um exemplo da abordagem proposta.

Tabela 1. Construção da expressão de busca para o refinamento da pesquisa.

\begin{tabular}{lc}
\hline \multicolumn{1}{c}{ Expressão de busca } & $\begin{array}{c}\text { Nr de } \\
\text { documentos }\end{array}$ \\
\hline (termo_1 AND termo_2) & Q1 \\
(termo_1 AND termo_2) OR (expressão_de_busca_original \\
$\begin{array}{l}\text { AND termo_1 AND termo_3) } \\
\text { (termo_1 AND termo_2) OR (termo_1 AND termo_3) OR } \\
\text { (termo_2 AND termo_4) }\end{array}$
\end{tabular}

\section{Exemplo de Aplicação da Abordagem Proposta}

A pesquisa bibliográfica foi realizada na base Scopus utilizando a expressão de busca "covid-19", que retornou 80.264 documentos publicados do período de 2019 a 2021 (fev). Neste resultado foram incluídos apenas os artigos publicados em periódicos, conferências e os do tipo revisão. Em seguida, fez-se um recorte na área de estudo das ciências sociais, que resultou em 10.965 documentos. Um segundo recorte foi realizado com a pesquisa do termo education, que resultou em 4.278 documentos.

Neste primeiro exemplo, foi escolhida a alternativa de analisar as arestas com os maiores pesos. Então, os metadados dos resultados da pesquisa foram salvos em formato CSV e importados pelo VOSviewer. A rede de coocorrência de palavras-chave, incluindo o tesauros para o controle do vocabulário, com pelo menos 2 ocorrências e cada comunidade com pelo menos 100 nós, foi gerada. Esta rede possui 1.770 nós, 6 comunidades e 10.319 arestas.

A rede foi salva em formato GML e importada pelo Gephi. Após calcular as métricas da rede de coocorrência de palavras-chave, foi selecionado o Laboratório de Dados e a opção de arestas. A Tabela 2 apresenta a seleção das 20 arestas com os maiores pesos. Como a rede é não direcionada, os nós de origem e destino não têm direção. Apenas representam as coocorrências das respectivas palavras-chave.

Em seguida, é construída a nova expressão de busca, que é apresentada na Tabela 3 , para a pesquisa bibliográfica na base Scopus. Para a construção da nova expressão de busca, as arestas $\mathrm{Nr} 04,09,10,12,15,18,19$ e 20 estão cobertas pela aresta 01 . As arestas $\mathrm{Nr}$ 08, 13, 14, 16 e 17 serão cobertas pela expressão: covid-19 AND "distance learning/self instruction". Como todos os pares de palavras-chave possuem o termo covid19, inicialmente foi feita a pesquisa usando este termo, seleção dos tipos de documentos da consulta inicial e período de 2.019 a 2.021. Em seguida, foram incluídas as expressões de busca listadas na Tabela 3 . O resultado alcançou a saturação na oitava iteração, com a recuperação de 4.244 documentos. 
Tabela 2. As 20 arestas com os maiores pesos.

\begin{tabular}{|c|c|c|c|}
\hline NR & Origem & Destino & Peso \\
\hline 01 & 1516 - covid-19 & 3719 - higher education & 140 \\
\hline 02 & 1516 - covid-19 & 5737 - online learning & 108 \\
\hline 03 & 1516 - covid-19 & 2162 - distance education & 93 \\
\hline 04 & 2173 - distance learning/self-instruction & 3719 - higher education & 67 \\
\hline 05 & 1516 - covid-19 & 2267 - e-learning & 54 \\
\hline 06 & 1516 - covid-19 & 5698 - online education & 45 \\
\hline 07 & 1516 - covid-19 & 7040 - remote education & 43 \\
\hline 08 & 2173 - distance learning/self-instruction & 9252 - web-based learning & 42 \\
\hline 09 & 3719 - higher education & 9252 - web-based learning & 34 \\
\hline 10 & 1723 - curriculum & 3719 - higher education & 33 \\
\hline 11 & 1516 - covid- 19 & 5789 - online teaching & 32 \\
\hline 12 & 3719 - higher education & 4578 - laboratory instruction & 32 \\
\hline 13 & 1723 - curriculum & 2173 - distance learning/self-instruction & 28 \\
\hline 14 & 2173 - distance learning/self-instruction & 4578 - laboratory instruction & 28 \\
\hline 15 & 3719 - higher education & 8179 - student-centered learning & 28 \\
\hline 16 & 2173 - distance learning/self-instruction & 3332 - general public & 27 \\
\hline 17 & 2173 - distance learning/self-instruction & 8179 - student-centered learning & 25 \\
\hline 18 & 2267 - e-learning & 3719 - higher education & 23 \\
\hline 19 & 2162 - distance education & 3719 - higher education & 22 \\
\hline 20 & 1289 - computer-based learning & 3719 - higher education & 21 \\
\hline
\end{tabular}

A construção da expressão de busca evidencia que o maior foco é na educação superior e nas modalidades de educação mediada pela tecnologia: educação a distância, aprendizado a distância, e-learning, educação on line, educação remota, ensino on line e auto-educação.

Tabela 3. Construção da expressão de busca para o refinamento da pesquisa.

\begin{tabular}{|c|c|}
\hline Expressão de busca & $\begin{array}{c}\mathrm{Nr} \text { de } \\
\text { documentos }\end{array}$ \\
\hline "higher education" & 2.768 \\
\hline "higher education" OR "online learning" & 3.338 \\
\hline "higher education" OR “online learning" OR "distance education" & 3.508 \\
\hline "higher education" OR "online learning" OR "distance education" OR e-learning & 3.960 \\
\hline $\begin{array}{l}\text { "higher education" OR "online learning" OR "distance education" OR e-learning OR } \\
\text { "online education" }\end{array}$ & 4.078 \\
\hline $\begin{array}{l}\text { "higher education" OR "online learning" OR "distance education" OR e-learning OR } \\
\text { "online education" OR "remote education" }\end{array}$ & 4.101 \\
\hline $\begin{array}{l}\text { "higher education" OR "online learning" OR "distance education" OR e-learning OR } \\
\text { "online education" OR "remote education" OR "distance learning/self-instruction" }\end{array}$ & 4.136 \\
\hline $\begin{array}{l}\text { "higher education" OR "online learning" OR "distance education" OR e-learning OR } \\
\text { "online education" OR "remote education" OR "distance learning/self-instruction" } \\
\text { OR "online teaching" }\end{array}$ & 4.244 \\
\hline
\end{tabular}


Os metadados da pesquisa foram salvos e lidos pelo VOSviewer. Foi gerada a rede de coocorrência de palavras-chave com o mínimo de 2 ocorrências, tesauros para o controle do vocabulário e cada cluster com o mínimo de 50 nós, resultando em 912 nós, 7 clusters e 4.703 arestas. A Figura 1 apresenta um mapa de densidade da rede de coocorrência de palavras-chave.

Como a palavra-chave covid-19 possui um grau alto, ela foi retirada para permitir a visualização de outros termos relevantes da pesquisa. Destacam-se as seguintes palavras-chave: higher education, distance education, online learning, distance learnimng/self-instruction, entre outras. A exploração da rede pode ser prosseguida com o cálculo das métricas de redes pelo Gephi e interpretação dos resultados.

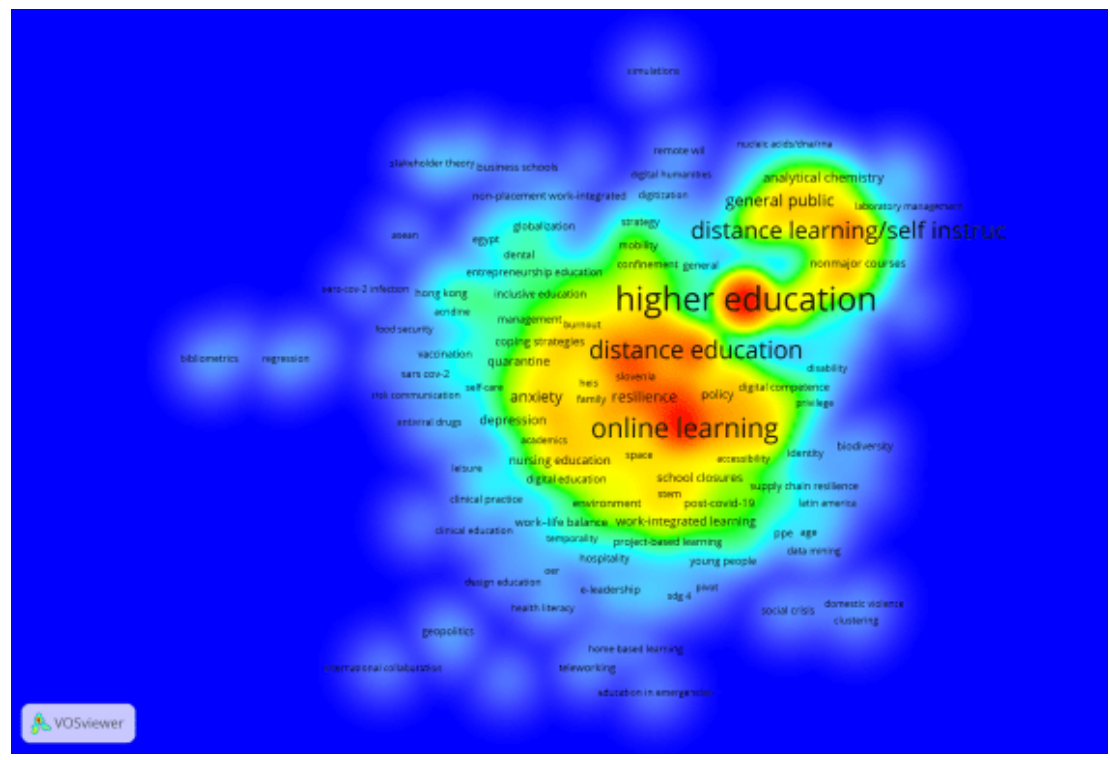

Fig. 1. Mapa de densidade da rede de coocorrência de palavras-chave.

A seguir, será mostrado um exemplo da segunda alternativa, onde a opção foi escolher um conceito representado por uma palavra-chave. Para este fim, foi escolhida a palavrachave higher education, por ter grande influência nas duas redes apresentadas anteriormente. Como a rede é não direcionada, foram selecionadas 271 arestas contendo a palavra-chave escolhida, na origem ou no destino. Em seguida, foram selecionadas as 16 arestas com maiores pesos. A primeira aresta é o foco da pesquisa e definirá o subconjunto de referências que será a base do refinamento. Nesta seleção foram retiradas arestas contendo palavras isoladas ou relativas a disciplinas como: analytical chemistry, biochemistry, applications of chemistry, chemical engineering, attitude, aromatic compounds, etc. A Tabela 4 apresenta as 16 arestas com os maiores pesos.

Como o tema central da pesquisa é Covid-19 e os pares de palavras-chave possuem o termo higher education, foi feita a primeira consulta usando o termo covid-19, seleção dos tipos de documentos da consulta inicial e período de 2.019 a 2.021. A pesquisa resultou em 80.264 documentos. Em seguida, foi feito um recorte com a palavra-chave higher education, que resultou em 2768 documentos, mesmo resultado da Tabela 3.

Para o refinamento, foi construída uma nova expressão de busca à semelhança do que foi feito anteriormente. A Tabela 5 apresenta a construção da expressão de busca com a combinação das palavras-chave da tabela anterior. A pesquisa alcançou a saturação na décima quinta iteração, com a recuperação de 1523 documentos. Como a variação foi pequena, a pesquisa poderia ter sido encerrada na décima segunda iteração.

Pode-se observar que as palavras-chave e-learning, distance education, blended learning, ethics e educational technology apresentaram um aumento mais significativo no número de documentos recuperados na Tabela 5 . 
Tabela 4. As 20 arestas com os maiores pesos.

\begin{tabular}{|c|c|c|c|}
\hline NR & Origem & Destino & Peso \\
\hline 01 & 3719 - higher education & 1516 - covid-19 & 140 \\
\hline 02 & 3719 - higher education & 2173 - distance learning/self-instruction & 67 \\
\hline 03 & 3719 - higher education & 2267 - e-learning & 23 \\
\hline 04 & 3719 - higher education & 2162 - distance education & 22 \\
\hline 05 & 3719 - higher education & 1289 - computer-based learning & 21 \\
\hline 06 & 3719 - higher education & 1115 - collaborative/cooperative learning & 17 \\
\hline 07 & 3719 - higher education & 3548 - hands-on learning/manipulative & 9 \\
\hline 08 & 3719 - higher education & 2066 - digital transformation & 6 \\
\hline 09 & 3719 - higher education & 2598 - emergency remote learning & 5 \\
\hline 10 & 3719 - higher education & 674 - blended learning & 4 \\
\hline 11 & 3719 - higher education & 2823 - ethics & 4 \\
\hline 12 & 3719 - higher education & 2600 - emergency remote teaching & 3 \\
\hline 13 & 3719 - higher education & 2487 - educational technology & 3 \\
\hline 14 & 3719 - higher education & 496 - augmented reality & 2 \\
\hline 15 & 3719 - higher education & 2460 - educational innovation & 2 \\
\hline 16 & 3719 - higher education & 1726 - curriculum design & 2 \\
\hline
\end{tabular}

Os metadados dos 1.523 documentos resultantes da pesquisa foram salvos e lidos pelo VOSviewer. Foi gerada a rede de coocorrência de palavras-chave com o mínimo de 2 ocorrências, tesauros para o controle do vocabulário e cada cluster com o mínimo de 40 nós, resultando em 600 nós, 6 clusters e 3.337 arestas.

Tabela 5. Construção da expressão de busca para o refinamento da pesquisa.

\begin{tabular}{|c|c|}
\hline Expressão de busca & $\begin{array}{c}\mathrm{Nr} \text { de } \\
\text { documentos }\end{array}$ \\
\hline "distance learning/self-instruction" & 53 \\
\hline "distance learning/self-instruction" OR e-learning & 950 \\
\hline "distance learning/self-instruction" OR e-learning OR "distance education" & 1.116 \\
\hline $\begin{array}{l}\text { "distance learning/self-instruction" OR e-learning OR "distance education" OR } \\
\text { "computer-based learning" }\end{array}$ & 1.121 \\
\hline $\begin{array}{l}\text { "distance learning/self-instruction" OR e-learning OR "distance education" OR } \\
\text { "computer-based learning" OR "collaborative/cooperative learning" }\end{array}$ & 1.123 \\
\hline $\begin{array}{l}\text { "distance learning/self-instruction" OR e-learning OR "distance education" OR } \\
\text { "computer-based learning" OR "collaborative/cooperative learning" OR "hands-on } \\
\text { learning/manipulative" }\end{array}$ & 1.123 \\
\hline $\begin{array}{l}\text { "distance learning/self-instruction" OR e-learning OR "distance education" OR } \\
\text { "computer-based learning" OR "collaborative/cooperative learning" OR "hands-on } \\
\text { learning/manipulative" OR "digital transformation" }\end{array}$ & 1.167 \\
\hline $\begin{array}{l}\text { "distance learning/self-instruction" OR e-learning OR "distance education" OR } \\
\text { "computer-based learning" OR "collaborative/cooperative learning" OR "hands-on } \\
\text { learning/manipulative" OR "digital transformation" OR "emergency remote learning" }\end{array}$ & 1.173 \\
\hline $\begin{array}{l}\text { "distance learning/self-instruction" OR e-learning OR "distance education" OR } \\
\text { "computer-based learning" OR "collaborative/cooperative learning" OR "hands-on } \\
\text { learning/manipulative" OR "digital transformation" OR "emergency remote learning" OR } \\
\text { "blended learning" }\end{array}$ & 1.231 \\
\hline $\begin{array}{l}\text { "distance learning/self-instruction" OR e-learning OR "distance education" OR } \\
\text { "computer-based learning" OR "collaborative/cooperative learning" OR "hands-on }\end{array}$ & 1.417 \\
\hline
\end{tabular}


learning/manipulative" OR "digital transformation" OR "emergency remote learning" OR "blended learning" OR ethics

"distance learning/self-instruction" OR e-learning OR "distance education" OR "computer-based learning" OR "collaborative/cooperative learning" OR "hands-on learning/manipulative" OR "digital transformation" OR "emergency remote learning" OR "blended learning" OR ethics OR "emergency remote teaching"

"distance learning/self-instruction" OR e-learning OR "distance education" OR "computer-based learning" OR "collaborative/cooperative learning" OR "hands-on learning/manipulative" OR "digital transformation" OR "emergency remote learning" OR "blended learning" OR ethics OR "emergency remote teaching" OR "educational technology"

"distance learning/self-instruction" OR e-learning OR "distance education" OR "computer-based learning" OR "collaborative/cooperative learning" OR "hands-on learning/manipulative" OR "digital transformation" OR "emergency remote learning" OR "blended learning" OR ethics OR "emergency remote teaching" OR "educational technology" OR "augmented reality"

"distance learning/self-instruction" OR e-learning OR "distance education" OR "computer-based learning" OR "collaborative/cooperative learning" OR "hands-on learning/manipulative" OR "digital transformation" OR "emergency remote learning" OR "blended learning" OR ethics OR "emergency remote teaching" OR "educational technology" OR "augmented reality" OR "educational innovation"

"distance learning/self-instruction" OR e-learning OR "distance education" OR "computer-based learning" OR "collaborative/cooperative learning" OR "hands-on learning/manipulative" OR "digital transformation" OR "emergency remote learning" OR "blended learning" OR ethics OR "emergency remote teaching" OR "educational technology" OR "augmented reality" OR "educational innovation" OR "curriculum design"

Os exemplos apresentados partiram de um tema atual que é a Covid-19 e analisou o espaço conceitual na área temática de Ciências Sociais, com um recorte em educação. Os exemplos revelaram que as modalidades de educação mediadas por tecnologia foram as mais relevantes. Como perspectivas futuras de pesquisa, sugere-se um aprofundamento na análise qualitativa dos artigos mais relevantes para identificar os aspectos que condicionaram estas opções e os respectivos resultados.

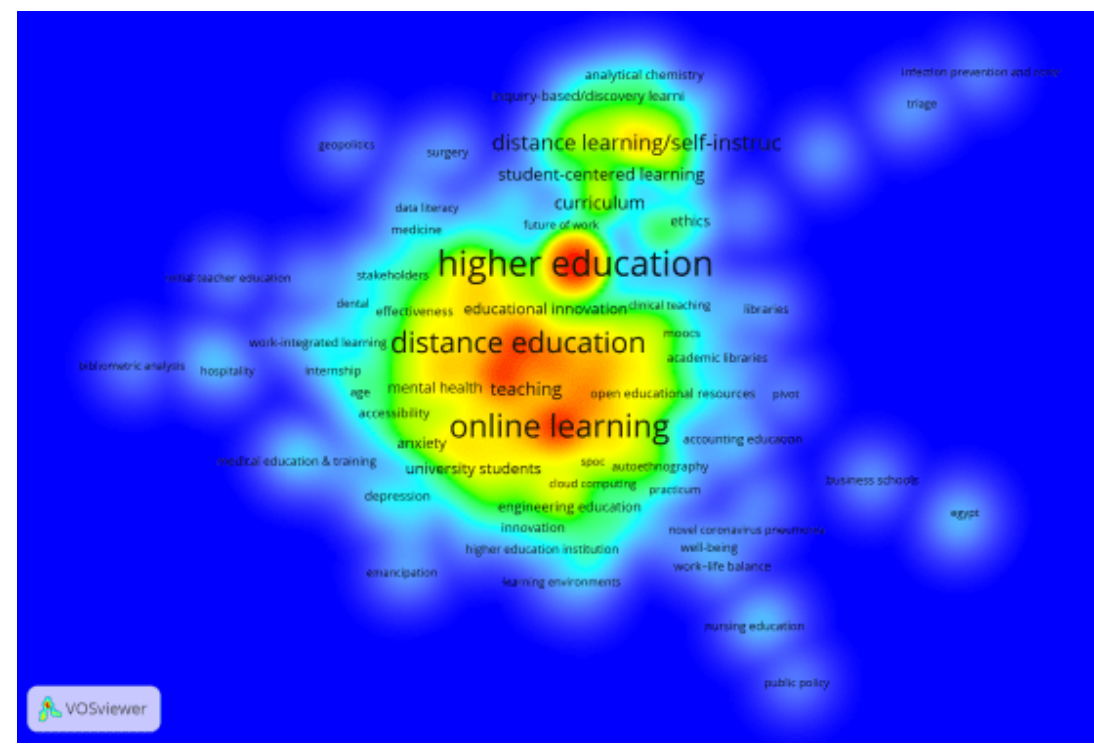

Fig. 2. Mapa de densidade da rede de coocorrência de palavras-chave. 
A Figura 2 apresenta um mapa de densidade da rede de coocorrência de palavras-chave. Como a palavra-chave covid-19 possui um grau alto, ela foi retirada para permitir a visualização de outros termos relevantes da pesquisa. Destacam-se as seguintes palavras-chave: higher education, distance education, online learning, e-learning, distance learnimng/self-instruction, entre outras. A exploração da rede pode ser prosseguida com o cálculo das métricas de redes pelo Gephi e interpretação dos resultados.

Os exemplos acima mostram caminhos possíveis para o refinamento de pesquisas bibliográficas. Outra possibilidade é a identificação de palavras-chave que podem delimitar um conceito. Por exemplo, a palavra-chave distance education pode compreender outros termos pertinentes ao conceito de educação a distância, tais como: online education, distance learning, online learning, distance learning/self-instruction, online teaching, distance teaching. Tudo dependerá da conceituação do tema e de sua delimitação para que o refinamento da pesquisa possa ser realizado com a análise das arestas de uma rede de coocorrência de palavras-chave.

\section{Conclusões}

Este artigo apresentou a proposta de uma abordagem para refinar uma pesquisa bibliográfica a partir da análise de uma rede de coocorrência de palavras-chave. Esta abordagem mostrou como identificar os pares de palavras-chave com maiores pesos na rede ou selecionar um determinado conceito e analisar as respetivas arestas.

Ao explorar um domínio temático, pode-se partir de uma pesquisa mais abrangente e empregar a rede de coocorrência de palavras-chave para identificar os conceitos relacionados pela análise dos nós e arestas. Ou seja, é uma alternativa e um complemento para estudos exploratórios sobre temas de pesquisa. A limitação inerente a termos selecionado só a base referencial Scopus pode ser ultrapassado por replicarmos este estudo exploratório para outras bases de dados como por exemplo WoS ou Scielo, para posteriormente observar as diferenças e semelhanças de modo a sintetizar uma visão geral sobre o tema em questão. Com os resultados obtidos, destes estudos a realizar no futuro, é possível apresentá-los a especialistas neste tema de modo a agregar valor a essa futura análise. Por outro lado será possível obter uma relevante e fulcral amostra de publicações que será objeto de análise de conteúdo que dará suporte a uma leitura crítica dessa literatura selecionada.

\section{Referências}

Bastian, M., Heymann, S., \& Jacomy, M. (2009). Gephi: An Open Source Software for Exploring and Manipulating Networks. In: Proceedings of the Third International ICWSM Conference, 361-362.

Besselaar, P., \& Heimeriks, G. (2006), Mapping research topics using word-reference cooccurrences: a method and an exploratory case study. Scientometrics, 68 (3), 377-393.

Börner, K., Chen, C., \& Boyack, K. (2003). Visualizing knowledge domains. Annual Review of Information Science and Technology, 37(1), 179-255.

Callon, M., Courtial, J.-P., Turner, W. A., \& Bauin, S. (1983). From translations to problematic networks: An introduction to co-word analysis. Social Science Information, 22(2), 191-235.

Choi, J., Yi, S., \& Lee, K. C. (2011). Analysis of keyword networks in MIS research and implications for predicting knowledge evolution. Information \& Management, 48(8), 371-381.

Coulter, N., Monarch, I., \& Konda, S. (1998). Software engineering as seen through its research literature: A study in co-word analysis. Journal of the American Society for Information Science, 49(13), 1206-1223.

Ding, Y., Chowdhury, G., \& Foo, S. (2001). Bibliometric cartography of information retrieval research by using co-word analysis", Information Processing and Management, 37 (6), 817. 
Gan, C., \& Wang, W. (2015). Research characteristics and status on social media in China: A bibliometric and co-word analysis. Scientometrics, 105(2), 1167-1182.

Khan, G. F., \& Wood, J. (2015). Information technology management domain: Emerging themes and keyword analysis. Scientometrics, 105(2), 959-972.

Kessler, M. M. (1963). Bibliographic coupling between scientific papers. American Documentation, 14 (1), 10-25.

Lee, T. S., Lee, Y. S., Lee, J., \& Chang, B. C. (2018). Analysis of the intellectual structure of human space exploration research using a bibliometric approach: Focus on human related factors. Acta Astronautica, 143, 169-182.

Luo, F., Dai, X, \& Huang, Y. (2021). The Analysis of Evolutionary Path of Research Topics on the Field of Visualization of International Cultural Heritage Information Since the 21st Century. In E3S Web of Conferences, 236, Art. 05074.

Marshakova, I. V. (1973). A system of document connection based on references. Scientific and Technical Information Serial of VINITI, 6 (2), 3-8.

Ravikumar, S., Agrahari, A., \& Singh, S. N. (2015). Mapping the intellectual structure of scientometrics: a co-word analysis of the journal Scientometrics (2005-2010). Scientometrics, $102(1), 929-955$

Small, H. (1973). Co-citation in scientific literature: a new measure of the relationship between publications. Journal of the American Society for Information Science, 24 (4), 265-269.

Van Eck, N. J., \& Waltman, L. (2021). VOSviewer manual. Leiden: Universiteit Leiden.

Wood, J., \& Khan, G. F. (2015). International trade negotiation analysis: Network and semantic knowledge infrastructure. Scientometrics, 105(1), 537-556.

Zhao, W., Mao, J., \& Lu, K. (2018). Ranking themes on co-word networks: Exploring the relationships among different metrics. Information Processing and Management, 54(2), 203218. 